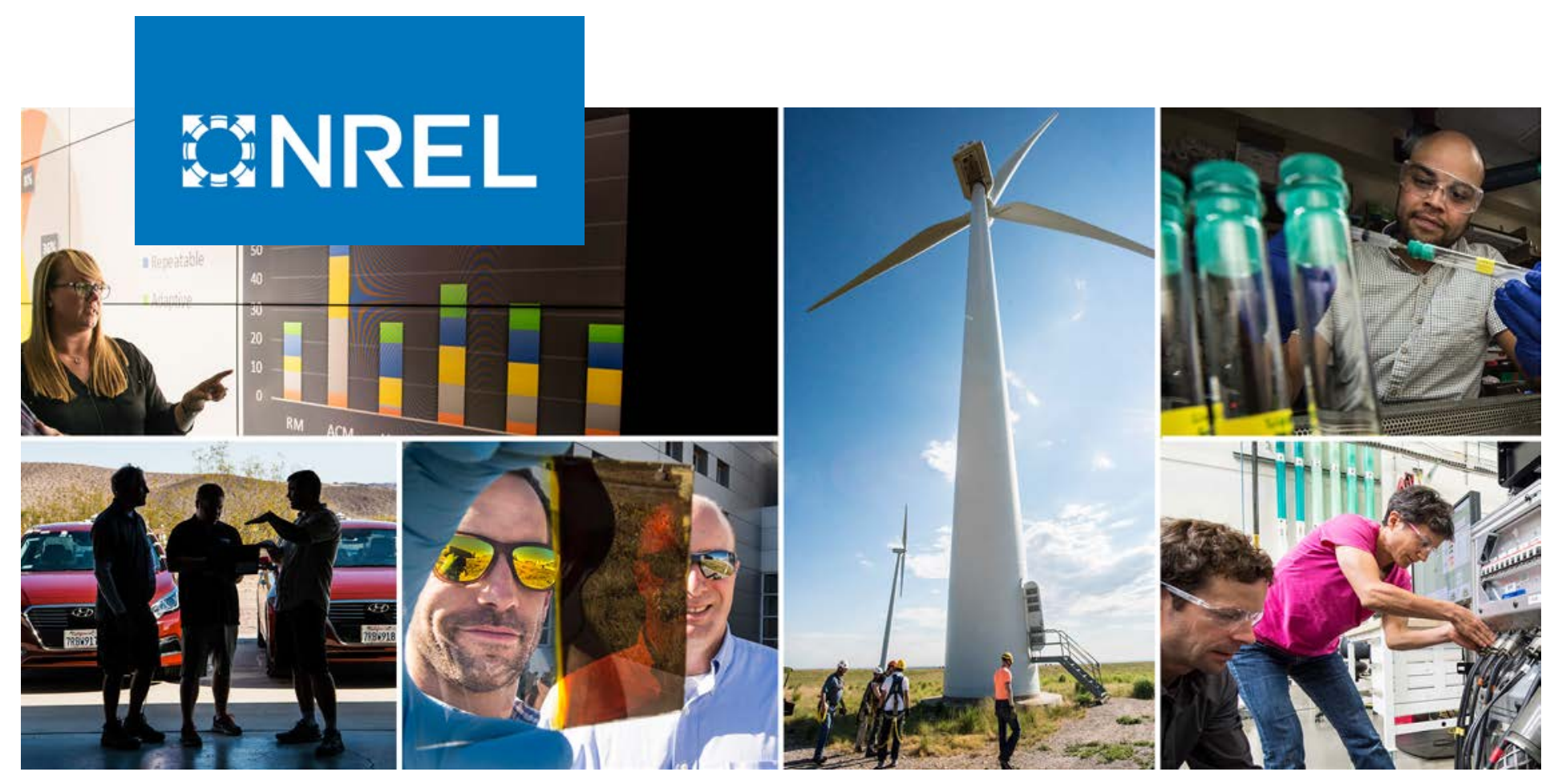

\title{
Linear Approximation Line Pack Model for Integrated Electricity and Natural Gas Systems OPF
}

\section{Preprint}

Xin Fang, Michael T. Craig, and Bri-Mathias Hodge

National Renewable Energy Laboratory

Presented at the IEEE Power and Energy Society (PES) General Meeting Atlanta, Georgia

August 4-8, 2019

NREL is a national laboratory of the U.S. Department of Energy

Office of Energy Efficiency \& Renewable Energy

Operated by the Alliance for Sustainable Energy, LLC

This report is available at no cost from the National Renewable Energy Laboratory (NREL) at www.nrel.gov/publications.

\section{Conference Paper}

NREL/CP-5D00-73763

August 2019 


\title{
BNREL
}

\section{Linear Approximation Line Pack Model for Integrated Electricity and Natural Gas Systems OPF}

\section{Preprint}

\author{
Xin Fang, Michael T. Craig, and Bri-Mathias Hodge \\ National Renewable Energy Laboratory
}

\author{
Suggested Citation \\ Fang, Xin, Michael T. Craig, and Bri-Mathias Hodge. 2019. Linear Approximation Line \\ Pack Model for Integrated Electricity and Natural Gas Systems OPF: Preprint. Golden, \\ CO: National Renewable Energy Laboratory. NREL/CP-5D00-73763. \\ https://www.nrel.gov/docs/fy19osti/73763.pdf.
}

(C) 2019 IEEE. Personal use of this material is permitted. Permission from IEEE must be obtained for all other uses, in any current or future media, including reprinting/republishing this material for advertising or promotional purposes, creating new collective works, for resale or redistribution to servers or lists, or reuse of any copyrighted component of this work in other works.

NREL is a national laboratory of the U.S. Department of Energy Office of Energy Efficiency \& Renewable Energy Operated by the Alliance for Sustainable Energy, LLC

This report is available at no cost from the National Renewable Energy Laboratory (NREL) at www.nrel.gov/publications.

Contract No. DE-AC36-08GO28308
Conference Paper

NREL/CP-5D00-73763

August 2019

National Renewable Energy Laboratory 15013 Denver West Parkway Golden, CO 80401

303-275-3000 • www.nrel.gov 


\section{NOTICE}

This work was authored by the National Renewable Energy Laboratory, operated by Alliance for Sustainable Energy, LLC, for the U.S. Department of Energy (DOE) under Contract No. DE-AC36-08GO28308. Funding provided U.S. Department of Energy Office of Energy Efficiency and Renewable Energy Wind Energy Technologies Office. The views expressed herein do not necessarily represent the views of the DOE or the U.S. Government. The U.S. Government retains and the publisher, by accepting the article for publication, acknowledges that the U.S. Government retains a nonexclusive, paid-up, irrevocable, worldwide license to publish or reproduce the published form of this work, or allow others to do so, for U.S. Government purposes.

This report is available at no cost from the National Renewable Energy Laboratory (NREL) at www.nrel.gov/publications.

U.S. Department of Energy (DOE) reports produced after 1991 and a growing number of pre-1991 documents are available free via www.OSTI.gov.

Cover Photos by Dennis Schroeder: (clockwise, left to right) NREL 51934, NREL 45897, NREL 42160, NREL 45891, NREL 48097, NREL 46526.

NREL prints on paper that contains recycled content. 


\title{
Linear Approximation Line Pack Model for Integrated Electricity and Natural Gas Systems OPF
}

\author{
Xin Fang, Michael T. Craig, Bri-Mathias Hodge \\ Power Systems Engineering Center \\ National Renewable Energy Laboratory \\ Golden, CO, USA 80401 \\ \{Xin.Fang, Michael.Craig, Bri.Mathias.Hodge\}@nrel.gov
}

\begin{abstract}
With the increasing installation of renewable energy electricity generation capacity, the penetration of natural gas fired generation is also substantailly increasing in power systems due to their fast response capability to mitigate system variability and uncertainty. The dependencies between the electricity system and the natural gas system are becoming more important to maintain the reliability of both systems. In integrated electricity and natural gas systems (IEGSs) operations, the nonlinearity of the natural gas system brings challenges to the system operators. In this paper, the IEGSs optimal power flow (OPF) model is proposed that includes the line pack of the gas system via linear approximation. The simulation results demonstrate that the proposed model can obtain optimal solutions efficiently that are comparable in accuracy as the nonlinear line pack model.
\end{abstract}

Index Terms-Integrated electricity and natural gas systems (IEGSs), optimal power flow, wind, reliability.

\section{NOMENCLATURE}

$t \quad$ Time index (hour)

S Number of gas wells

$\mathrm{T} \quad$ Time span of the model

C Number of compressors (index $c$ )

PL Number of pipelines (index $p l$ )

GL Number of residential gas loads (index $g l$ )

$\mathrm{L} \quad$ Number of transmission lines (index $l$ )

$\mathrm{N} \quad$ Number of buses in electricity system (index $i$ )

M Number of generation units (index $i$ )

W Number of wind power units (index $i$ )

$c_{i, 0}, c_{i, 1}, c_{i, 2}$ Gas consumption coefficients of generator at

$c_{i, 0}, c_{i, 1}, c_{i, 2}$ bus $i(\mathrm{Gj} / \mathrm{MWh})$

$\rho_{i, g a s} \quad$ Fuel price for generator unit $i(\$ / \mathrm{Gj})$

$F_{i, t} \quad$ Generator at bus $i$ gas consumption $(\mathrm{Gj})$

$R_{c}^{\min } / R_{c}^{\max }$ Compressor's ratio minimum/maximum limits

$F C_{c}^{\max } \quad$ Maximum flow limit in compressors

$\boldsymbol{S}_{\boldsymbol{w}} \quad$ Gas supply matrix from the gas well $(\mathrm{S} \times \mathrm{T})$

$\boldsymbol{G D}_{\boldsymbol{d}} \quad$ Residential gas consumption matrix $(\mathrm{GL} \times 1)$

$\boldsymbol{F} \boldsymbol{L}_{\boldsymbol{l}} \quad$ Gas flow matrix in pipelines $(\mathrm{PL} \times \mathrm{T})$

$\boldsymbol{L P}_{\boldsymbol{l}} \quad$ Line pack matrix in pipelines $(\mathrm{PL} \times \mathrm{T})$

$\boldsymbol{F C}_{\boldsymbol{c}} \quad$ Gas flow matrix in compressors $(\mathrm{C} \times \mathrm{T})$

$R_{c, t} \quad$ Compression ratio of compressor $c$ at time $t$
$S_{w, t}^{\min } / S_{w, t}^{\max }$ Gas wells production upper/lower limits

$p_{n, t}^{\max } / p_{n, t}^{\min }$ Gas node pressure maximum $/$ minimum limits at the $n$-th gas node at time $t$

$p_{n, t} \quad$ Gas node pressure at the $n$-th gas node at time $t$

$H_{c, t} \quad$ Power consumption of Compressor $c$ at time $t$

$K_{m n} \quad$ Pipeline parameter for gas flow from $m$ to $n$

$C_{m n} \quad$ Pipeline parameter for line pack from $m$ to $n$

$G_{i, t} \quad$ Generation power output at bus $i$ (MW)

$G_{i}^{\max } / G_{i}^{\min }$ Upper/lower generation level at bus $i$ (MW)

$D_{i, t} \quad$ Demand quantity (MW) at bus $i$ at time $\mathrm{t}$

$P_{i, t} \quad$ Wind power output (MW) at bus i at time t

$\boldsymbol{G S F} \quad$ Generation shift factor matrix $(\mathrm{L} \times \mathrm{N})$

$\boldsymbol{L} \boldsymbol{U} \quad$ Line limit $(\mathrm{L} \times 1)$

$R_{i}^{U} / R_{i}^{D} \quad$ Ramp-up/-down capability (MW/minutes) of

$\Delta t \quad$ Length of the time interval (minutes)

All the other variables are explained in the manuscript text.

\section{INTRODUCTION}

Recently, the penetration of natural gas fired generation units has substantially increased in the United States power system because of low gas prices and the reduced emissions compared to coal generators [1], [2]. Many of these gas units have the capability to change their power output levels rapidly and are therefore often utilized to mitigate the system variability and uncertainty of load and variable renewable generation [3], [4]. In several power systems managed by independent system operators (ISOs), such as PJM, ERCOT, and CAISO, the generation capacity of natural gas generation is already over a $40 \%$ share, as shown in Fig. 1. Because of this increasing reliance on natural gas for power production, the dependency between the electricity system and natural gas system should be taken into consideration in both system's operations to maintain their reliability. The integrated electricity and natural gas systems (IEGSs) operation is being proposed to deal with the dependency between two systems.

Recently, there have been many contributions to the literature on the co-simulation/co-optimization of the IEGSs. In [5], the uncertainty of wind power output was modeled in the 
co-optimization of IEGSs. The intervals of power system operating cost and gas system operating parameters such as nodes pressure were obtained considering the wind power output uncertainty variation. In [6], the generation offering decision was obtained considering the uncertainties in both the electricity system and the natural gas system. The IEGSs simulation considering the nonlinearity of the natural gas system was investigated in [7], [8]. A linear IEGSs model for electricity market clearing was presented in [9]. In these studies, the gas network line pack, which is an inherent storage capability of the gas pipelines, was either ignored or modeled with a nonlinear formulation. The natural gas line pack storage capacity can mitigate gas shortages and provide additional flexibility to the IEGSs, especially under a high renewable penetration level. Therefore, to accurately model the line pack in the IEGSs simulation is an urgent and important issue. In this paper, the IEGSs optimal power flow (OPF) is proposed considering the impact of gas network line pack. A linear approximation model is used to model the line pack which is efficient to solve and maintains a high accuracy compared to the nonlinear model.

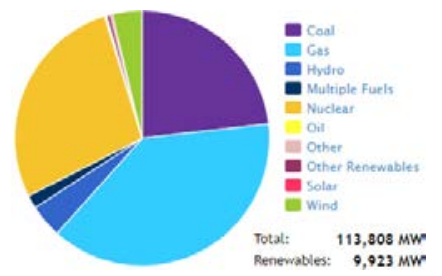

Fig. 1. Generation fuel mix in PJM [10]

The rest of this paper is organized as follows. Section II proposes the linear approximation of the natural gas line pack model and the co-optimization model of IEGSs OPF. Section III performs case studies to illustrate the impact of different gas network line pack modeling and the influence of wind power penetration. Finally, Section IV concludes the paper.

\section{IEGSS OPF WITH LINEAR LINE PACK APPROXIMATION}

\section{A. Linearized Average Gas Pressure for Line pack}

In the gas network line pack modelling, the average pressure in a pipeline should be formulated. In the previous research, this average pressure was modelled with the mean or a nonlinear relation of in-flow and out-flow node pressures [7], [11]. Using the mean might lead to significant errors when the pressure difference between the in-flow and out-flow nodes is large. Using the nonlinear formulation brings the computation burden to the IEGSs optimization when the network is large. In this paper, a linear approximation is introduced to capture the nonlinear relationship of the average pressure in a pipeline with in-flow and out-flow node pressures, while maintaining the computational efficiency of a linear model.

First, the pressure in a pipeline is quadratically related with the in-flow and out-flow nodes pressure shown in Fig. 2.

$$
p_{x}=\sqrt{p_{1}^{2}-\left(p_{1}^{2}-p_{2}^{2}\right) \cdot \frac{x}{L}}
$$

where $p_{x}$ is the pressure at the location $x ; x$ is the distance to the in-flow node; $L$ is the pipeline length.

Then, the average pressure in a pipeline is shown in Eq. (2).

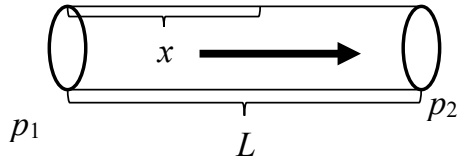

Fig. 2. Illustration of gas pressure in a pipeline

$$
\begin{aligned}
\tilde{p}_{p l-m n, t}= & \int_{0}^{L} \frac{1}{L} \sqrt{p_{1}^{2}-\left(p_{1}^{2}-p_{2}^{2}\right) \cdot \frac{x}{L}} d x= \\
& \frac{2}{3} \frac{p_{1}^{2}+p_{1} p_{2}+p_{2}^{2}}{p_{1}+p_{2}}=\frac{2}{3}\left(p_{1}+p_{2}-\frac{p_{1} p_{2}}{p_{1}+p_{2}}\right)
\end{aligned}
$$

The out-flow node pressure can be represented as in Eq. (3).

$$
\begin{aligned}
& p_{2}=r_{1,2} p_{1} \\
& \frac{p_{1} p_{2}}{p_{1}+p_{2}}=\frac{r_{1,2}}{1+r_{1,2}} p_{1}=k_{1,2} p_{1}
\end{aligned}
$$

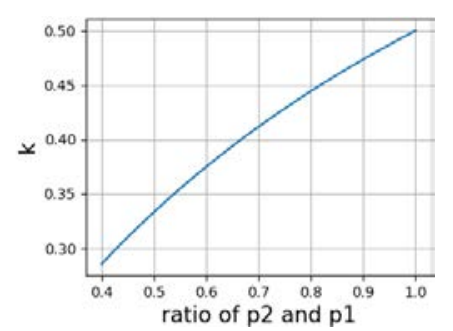

Fig. 3. Illustration of $\mathrm{k}$ with ratio of $\mathrm{p} 2$ and $\mathrm{p} 1$

From Eq. (2), using the mean $\frac{1}{2}\left(p_{1}+p_{2}\right)$ to represent the average pressure in the pipeline leads to inaccurate results when the pressure difference is high.

In a pipeline, the in-flow node gas pressure is higher than the out-flow node $\left(r_{1,2}<1\right)$ to maintain appropriate system pressure. From Fig. 3, an appropriate constant $k$ in Eq. (4) can be chosen to linearize Eq. (2). The procedure of the iterative linear approximation for the average pipeline gas pressure is:

Step 1: The constant $k$ is chosen for each pipeline initially according to their nodal pressure differences (or with a flat start, these constants are set to 0.5 initially);

Step 2: After solving the IEGSs OPF model, the constants are updated for every pipeline at every time interval with the updated in-flow and out-flow node gas pressures.

Step 3: The model is solved again with updated $k$ values.

Step 4: The iteration stops when the difference of the objective function between two iterations is smaller than the predefined threshold value.

The values of $k$ is updated from Step 1 to Step 4, and the gas line pack model is linearized.

\section{B. IEGSs OPF Model}

In this IEGSs OPF study, the total generation cost in the electricity system is minimized while maintaining all operating parameters in IEGSs within their limits during different intervals. The IEGSs OPF model is presented in Eqs. (5) to (21). $\min \sum_{t \in T}\left(\sum_{i \in g} \rho_{i, g a s}\left(c_{i, 2} G_{i, t}^{2}+c_{i, 1} G_{i, t}+c_{i, 0}\right)\right)$

$\boldsymbol{G}_{\boldsymbol{t}}-\boldsymbol{G}_{\boldsymbol{t}-\mathbf{1}} \leq \boldsymbol{R}^{\boldsymbol{U}} \cdot \Delta t$

$\boldsymbol{G}_{t-1}-\boldsymbol{G}_{t} \leq \boldsymbol{R}^{D} \cdot \Delta t$

$G^{\text {min }} \leq G_{t} \leq G^{\text {max }}$

$I_{G} \times G_{t}+I_{W} \times P_{t}-I_{D} \times D_{t}=0$

$-\boldsymbol{L U} \leq \boldsymbol{G S F} \times\left(\boldsymbol{G}_{\boldsymbol{t}}+\boldsymbol{P}_{\boldsymbol{t}}-\boldsymbol{D}_{\boldsymbol{t}}\right) \leq \boldsymbol{L U}$

$F_{i, t}=c_{i, 2} G_{i, t}^{2}+c_{i, 1} G_{i, t}+c_{i, 0}$ 
$I_{w} S_{w}-I_{d} G D_{g l}+I_{l-t} F L_{p l-i n}+I_{c-t} F C_{c}-I_{l-f} F L_{p l-o u t}-$

$\boldsymbol{I}_{\boldsymbol{c}-\boldsymbol{f}} \boldsymbol{F} \boldsymbol{C}_{\boldsymbol{c}}-\boldsymbol{I}_{\boldsymbol{g}} \boldsymbol{F}=\mathbf{0}$

$S_{w, t}^{\min } \leq S_{w, t} \leq S_{w, t}^{\max }$

$F L_{p l, t} \leq K_{m n} \frac{P I_{m n, s}}{\sqrt{P I_{m n, s}^{2}-P O_{m n, s}^{2}}} p_{m, t}-K_{m n} \frac{P O_{m n, s}}{\sqrt{P I_{m n, s}^{2}-P O_{m n, s}^{2}}} p_{n, t}$

$F C_{c, t}=-\frac{H_{c, 0}}{k_{c, 2}-k_{c, 1} R_{0}^{\alpha_{c}}}+\frac{\partial F_{c, t}}{\partial H_{c, t}} \times\left(H_{c, t}-H_{c, 0}\right)+\frac{\partial F_{c, t}}{\partial p_{m, t}} \times$

$\left(p_{m, t}-p_{m 0}\right)+\frac{\partial F_{c, t}}{\partial p_{n, t}} \times\left(p_{n, t}-p_{n 0}\right)$

$R_{c}^{\text {min }} \leq R_{c, t}=\frac{p_{n, t}}{p_{m, t}} \leq R_{c}^{\max }$

$p_{n, t}^{\min } \leq p_{n, t} \leq p_{n, t}^{\max }$

$F C_{c, t} \leq F C_{c}^{\max }$

$F L_{p l, t}=\left(F L_{p l-i n, t}+F L_{p l-o u t, t}\right) / 2$

$L P_{p l, t}=C_{m n} \tilde{p}_{p l-m n, t}$

$L P_{p l, t}=L P_{p l, t-1}+F L_{p l-i n, t}-F L_{p l-o u t, t}$

where $\boldsymbol{I}_{\boldsymbol{G}}, \boldsymbol{I}_{\boldsymbol{W}}, \boldsymbol{I}_{\boldsymbol{D}}$ are the incidence matrices of conventional generators, wind power plants and electricity load to the electricity system buses; $\boldsymbol{I}_{\boldsymbol{w}}, \boldsymbol{I}_{\boldsymbol{d}}, \boldsymbol{I}_{\boldsymbol{l}-\boldsymbol{t}}, \boldsymbol{I}_{\boldsymbol{c}-\boldsymbol{t}}, \boldsymbol{I}_{\boldsymbol{l}-\boldsymbol{f}}, \boldsymbol{I}_{\boldsymbol{c}-\boldsymbol{f}}$, and $\boldsymbol{I}_{\boldsymbol{g}}$ are the incidence matrices of gas wells, natural gas loads, gas pipelines "to" nodes, compressors "to" nodes, gas pipelines "from" nodes, compressors "from" nodes, and gas-fired generator units to the natural gas network nodes; $\alpha_{c}$ in Eq. (15) is a constant determined by the polytropic exponent of the compressor; and $k_{c, 1}$ and $k_{c, 2}$ in Eq. (15) are empirical parameters associated with the compressor design. Eq. (5) gives the quadratic generation cost which is represented by a set of linearized segments; (6)-(8) are generation limits in the electricity system; Eq. (9) is the power balance equation of the electricity system; (10) is the transmission power flow limits; Eq. (11) represents the gas consumption of gas-fired generator units; Eq. (12) shows the nodal gas balance equation of the natural gas network; (13) is the gas well production limits; Eq. (14) and (15) are the linearized gas flows equations in pipelines and compressors; (16) limits the compressor's compression ratio; (17) shows the nodal gas pressure limitation; (18) shows the compressor's gas flow limit; (19) shows that the pipeline gas flow is the average of its in-flow and out-flow; (20) and (21) are the pipeline line pack equations. $\tilde{p}_{p l-m n, t}$ is the average gas pressure in the pipeline $m n$ which is calculated using the linear approximation in the previous subsection. The original nonlinear formulations of Eq. (14) and (15) and the procedure for their linearization are listed below for the model completeness.

The natural gas flow through a pipeline depends on the pipeline parameters (diameter, fiction coefficients, etc) and the pressure difference between the in-flow and out-flow nodes. The nonlinear Weymouth equation is used to model the turbulent gas flow in all pipelines [12], [13] as:

$F L_{p l, t}\left(p_{m, t}, p_{n, t}\right)=K_{m n} \sqrt{p_{m}^{2}-p_{n}^{2}}$

To linearize the nonlinear equation in (22), first the first order Taylor expansion of the non-linear Weymouth Eq. (22) at the given values of $P I$ and $P O$ which are the in-flow and outflow nodes pressures is shown in Eq. (23).

$$
\begin{aligned}
& F L_{p l, t}\left(p_{m, t}, p_{n, t}\right)=F L_{p l, t}\left(P I_{m n}, P O_{m n}\right)+\frac{\partial F L_{p l, t}}{\partial p_{m}}\left(p_{m}-\right. \\
& \left.P I_{m n}\right)+\frac{\partial F L_{p l, t}}{\partial p_{n}}\left(p_{n}-P O_{m n}\right)
\end{aligned}
$$

Then introducing a set of points $\left(P I_{m n s}, P O_{m n s}\right)$ to linearize the Taylor expansion in Eq. (23), where $s \in\{1,2, \cdots, S\}$ is the index of the linearization segments. Then the nonlinear equation in Eq. (23) can be replace by a set of linearized inequality constraints in (24). More details about this linearization can be found in [6] and [14].

$$
\begin{gathered}
F L_{p l, t}\left(p_{m, t}, p_{n, t}\right) \leq K_{m n} \frac{P I_{m n, s}}{\sqrt{P I_{m n, s}^{2}-P O_{m n, s}^{2}}} p_{m, t}- \\
K_{m n} \frac{P O_{m n, s}}{\sqrt{P I_{m n, s}^{2}-P O_{m n, s}^{2}}} p_{n, t}
\end{gathered}
$$

The gas flow in a compressor is determined by the compressor's power consumption and the compression ratio shown in Eq. (25).

$$
F C_{c, t}\left(H_{c, t}, R_{c, t}\right)=-\frac{H_{c, t}}{k_{c, 2}-k_{c, 1}\left(R_{c, t}\right)^{\alpha_{c}}}
$$

Similarly, the gas flow in the compressors are also linearized with its first order Taylor expansion at a fixed operating point $\left(H_{c 0}, p_{m 0}, p_{n 0}\right)$. The initial compression ratio is $R_{c 0}=\frac{p_{n 0}}{p_{m 0}}$. The linearized formulation of Eq. (26) is as,

$$
\begin{gathered}
F C_{c, t}\left(H_{c, t}, R_{c, t}\right)=-\frac{H_{c, 0}}{k_{c, 2}-k_{c, 1} R_{0}^{\alpha_{c}}}+\frac{\partial F_{c, t}}{\partial H_{c, t}} \times\left(H_{c, t}-H_{c, 0}\right)+ \\
\frac{\partial F_{c, t}}{\partial p_{m, t}} \times\left(p_{m, t}-p_{m 0}\right)+\frac{\partial F_{c, t}}{\partial p_{n, t}} \times\left(p_{n, t}-p_{n 0}\right)
\end{gathered}
$$

\section{CASE STUdies}

\section{A. Six-Bus Electricity Network with Seven-Node Natural Gas Network}

A small IEGSs consisting of a six-bus electricity system and a seven-node natural gas network is depicted in Fig. 4, and the system parameters are from [6], [13]. Five generation units are connected to buses 1,2 and 6 in which two units are gas-fired units. Two $35 \mathrm{MW}$ wind power plants are connected to buses 3 and 5.

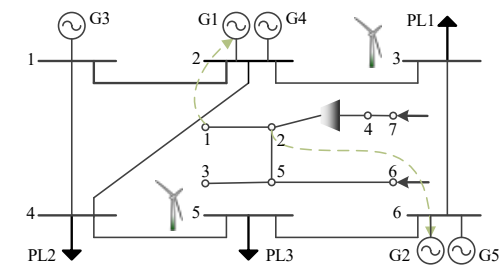

Fig. 4. Six-bus system and generation parameters

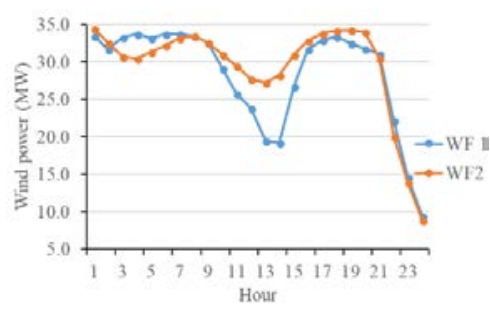

Fig. 5. Demand and wind power curves

The 24 hour electricity and natural gas residential loads, and the parameters of the natural gas system can be found in Appendix $\mathrm{C}$ of [6]. The wind power forecasts are shown in Fig. 5; they are from the Wind Toolkit [15] with plant identification 
numbers 46851 and 46324 . The forecasted wind power is scaled up to $35 \mathrm{MW}$ for each wind power plant. In this study, it assumed that the gas well 1 and 2 have firm contracts to produce $5000 \mathrm{Mcm} / \mathrm{hr}$ and $6000 \mathrm{Mcm} / \mathrm{hr}$ gas, respectively.

\section{B. Reliability Impact of IEGSs Coordination}

This subsection studies the reliability impact of the coordiation between the electricity and natural gas networks. Five cases of OPF with different IEGSs constraints modeling are performed. Five cases are shown below. The nonlinear line pack modeling in Case 3 will be chosen as a benchmark model to validate the accuracy of the proposed model because the nonlinear formulation accurately models the gas network. The system operating costs and the gas consumption of five cases are listed in Table 1. Fig. 6 to Fig. 9 are the node pressue and pipelines' line pack under different cases.

Case 1: OPF of only electricity network;

Case 2: OPF of IEGSs with gas line pack model using mean as the pipeline average pressure;

Case 3: OPF of IEGSs with gas line pack model using nonlinear average pressure model shown in Eq. (2);

Case 4: OPF of IEGSs with gas line pack model using proposed average pressure model.

Table 1 System operating cost and gas consumption in four cases

\begin{tabular}{ccccc}
\hline \hline & Case 1 & Case 2 & Case 3 & Case 4 \\
\hline System cost (\$) & $2,426,980$ & $2,547,078$ & $2,547,396$ & $2,547,405$ \\
\hline Gas consumption $(\mathrm{Mcm})$ & $288,920.4$ & 264,000 & 264,000 & 264,000 \\
\hline \hline
\end{tabular}

Table 1 shows that without considering the constraints of natural gas network the system operating cost is lowest shown in Case 1. However, the solution in Case 1 is not physically feasible because the gas production and node pressure violate their limits in this solution shown in Table 1 and Fig. 6.

Comparing the results among Case 2 to Case 4, it demonstrates that the proposed linear approximation model in Case 4 can obtain a very accurate results compared to the nonlinear model in Case 3 with the operating cost difference less than $0.00035 \%$. While using the mean to represent the average gas pressure in Case 2 leads to $0.012 \%$ error in the operating cost compared to Case 3. Therefore, the proposed model obtains a more accurate results compared to the model using the mean as the average gas pressure in the pipeline.

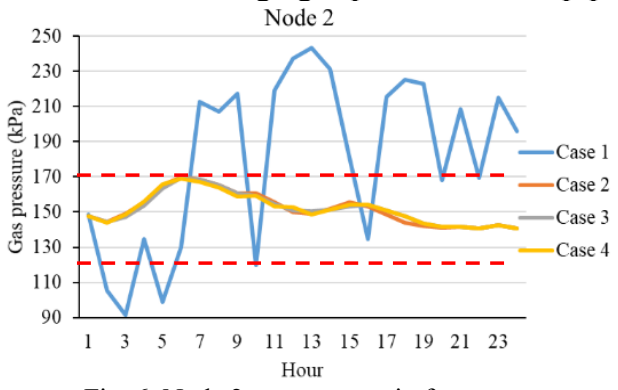

Fig. 6. Node 2 gas pressure in four cases

Fig. 6 and Fig. 7 demonstrate the gas pressure of node 2 and 3 in different cases. The red dash lines are the lower and upper limits of the node pressure. Fig. 6 shows that without considering the gas network constraints the electricity system dispatch leads to large violations in the gas network. This means that the solution is not physically feasible. After considering the gas network limitations in Case 2 to Case 4, the node pressure can be maintained within its limits. Fig. 7 shows that the pressures of node 3 in Case 2 to Case 4 are close.

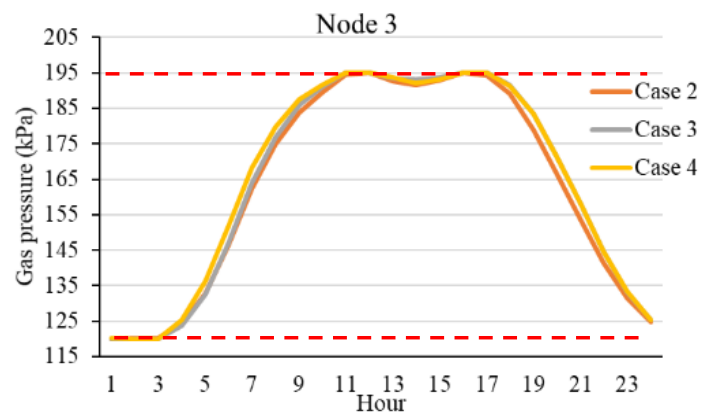

Fig. 7. Node 3 gas pressure in Case 2, Case 3, and Case 4

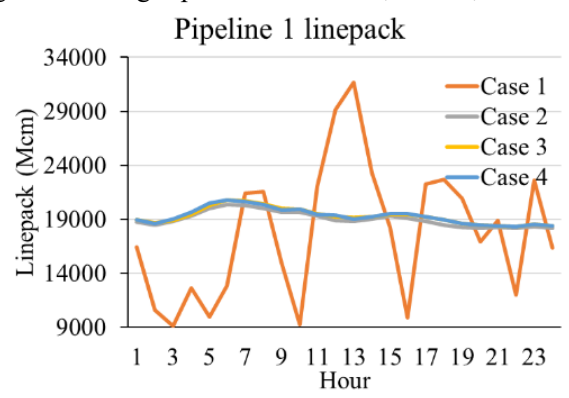

Fig. 8. Line pack of pipeline 1 in four cases

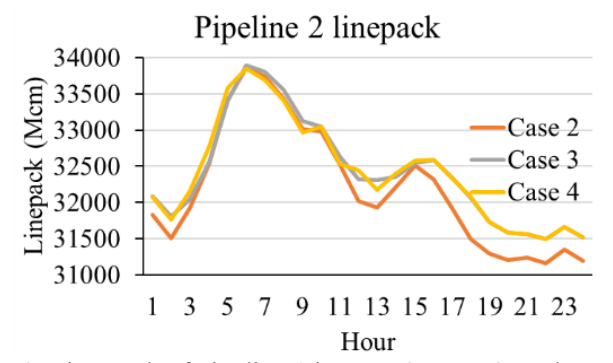

Fig. 9. Line pack of pipeline 1 in Case 2, Case 3, and Case 4

Fig. 8 and Fig. 9 show the 24 hours line pack of pipeline 1 and 2 in different cases. Fig. 8 demonstrates that without a cooptimization modeling the electricity system solution leads to a large line pack variation in the gas network which is also physically infeasible. Case 2 to Case 4 can obtain a physically feasible solutions for the IEGSs after considering the dependency between two systems. Fig. 9 shows that the proposed model can obtain the line pack results close to the nonlinear accurate model. Using the mean to represent the average pressure in Case 3 leads to a large deviation in the line pack results.

\section{Wind Power Penetration}

The comparison among Case 2 to Case 4 under different wind power penetration levels is investigated in this subsection. In this study, the wind penetration is adjusted with a scale $k p$. For instance, when the value of $k p$ is 1.1, the wind power output in Fig. 5 is multiplied by 1.1. The system operating costs under different penetration levels are listed in Table 2 for Case 2 to Case 4.

From Table 2, it is obvious that the proposed model can obtain the results close to the benchmark nonlinear line pack 
model regardless of the penetration levels. However, with the mean to represent the average pipeline gas pressure in Case 2, the operating cost deviation between Case 2 and Case 3 is large especially when the wind penetration level is high.

Table 2 System operating cost in three cases under different wind penetration levels

\begin{tabular}{cccc}
\hline \hline$k p$ & Case 2 & Case 3 & Case 4 \\
\hline 1.0 & $2,547,078$ & $2,547,396$ & $2,547,405$ \\
\hline 1.1 & $2,482,871$ & $2,483,189$ & $2,483,268$ \\
\hline 1.2 & $2,418,824$ & $2,419,151$ & $2,419,210$ \\
\hline 1.3 & $2,354,866$ & $2,355,195$ & $2,355,218$ \\
\hline 1.4 & $2,291,051$ & $2,291,397$ & $2,291,400$ \\
\hline 1.5 & $2,227,391$ & $2,227,738$ & $2,227,752$ \\
\hline \hline
\end{tabular}

\section{Computational Efficiency}

All the case studies are performed on a Dell Laptop with Intel Core i5 as the CPU. The proposed iterative linear approximation model can obtain the optimal solutions within 6 iterations in less than 2 seconds for the previous case studies. Therefore, it is efficient to obtain the solution accurately and close to the results of the nonlinear model. Note that for a large natural gas system, the global optimum cannot be guaranteed using the nonlinear model. While with the proposed model, the optimal solution can be obtained.

\section{CONCLUSIONS}

This paper proposes a linear approximation model for the natural gas line pack modeling in the IEGSs OPF problem. The nonlinear gas network constraints are linearized and can be efficiently solved. The case studies show that the dependency between electricity and natural gas systems should be modeled to maintain a reliable system operation. The simulation results demonstrate that the proposed model can obtain accurate optimal solutions compared to the nonlinear line pack model. Using the mean of in-flow and out-flow node pressure in the line pack model leads to inaccurate results especially when the renewable penetration is high.

\section{ACKNOWLEDGEMENTS}

This work was authored by Alliance for Sustainable Energy, LLC, the Manager and Operator of the National Renewable Energy Laboratory for the U.S. Department of Energy (DOE) under Contract No. DE-AC36-08GO28308. Funding provided by U.S. Department of Energy Office of Energy Efficiency and Renewable Energy Wind Energy Technologies Office. The views expressed in the article do not necessarily represent the views of the DOE or the U.S. Government. The U.S. Government retains and the publisher, by accepting the article for publication, acknowledges that the U.S. Government retains a nonexclusive, paid-up, irrevocable, worldwide license to publish or reproduce the published form of this work, or allow others to do so, for U.S. Government purposes.

\section{REFERENCES}

[1] C. Liu, C. Lee, and M. Shahidehpour, "Look ahead robust scheduling of wind-thermal system with considering natural gas congestion," IEEE Trans. Power Systems, vol. 30, no. 1, pp. 544-545, 2015.
[2] X. Fang, L. Bai, F. F. Li, et al, "Hybrid Component and Configuration Model for Combined-Cycle Units in the Unit Commitment Problem," Journal of Modern Power Systems and Clean Energy, pp. 1-6, 2018.

[3] C. He, L. Wu, et al, "Robust Co-Optimization Scheduling of Electricity and Natural Gas Systems via ADMM," IEEE Trans. Sustain. Ener., vol. 8, no. 2, 2017.

[4] X. Fang, B. Hodge, H. Jiang, and Y. Zhang, "Decentralized wind uncertainty management: Alternating direction method of multipliers based distributionally-robust chance constrained optimal power flow," Applied Energy, vol. 239, pp. 938-947, 2019.

[5] L. Bai, F. Li, H. Cui, et al, "Interval optimization based operating strategy for gas-electricity integrated energy systems considering demand response and wind uncertainty," Applied Energy, vol. 167, 2016.

[6] H. Cui, F. Li, et al, "Day-ahead coordinated operation of utility-scale electricity and natural gas networks considering demand response based virtual power plants," Applied Energy, vol. 176, no. 15, pp. 183-195, 2016.

[7] K. A. Pambour, B. Cakir Erdener, et al, "SAInt - A novel quasi-dynamic model for assessing security of supply in coupled gas and electricity transmission networks," Applied Energy, vol. 203, pp. 829-857, 2017.

[8] J. Fang, Q. Zeng, et al, "Dynamic optimal energy flow in the integrated natural gas and electrical power systems," IEEE Trans. Sustain. Ener., vol. 9, no. 1, 2018.

[9] C. Ordoudis, P. Pinson, et al, "An Integrated Market for Electricity and Natural Gas Systems with Stochastic Power Producers," European Journal of Operational Research, vol. 272, no. 2, pp. 642-654, 2018.

[10] "PJM Generation fuel mix." [Online]. Available: https://www.pjm.com/markets-and-operations.aspx.

[11] C. M. Correa-Posada and P. Sanchez-Martin, "Integrated Power and Natural Gas Model for Energy Adequacy in Short-Term Operation," IEEE Transactions on Power Systems, vol. 30, no. 6, pp. 3347-3355, 2015.

[12] K. T. Midthun, Optimization models for liberalized natural gas markets, no. October. 2007.

[13] C. Liu, M. Shahidehpour, Y. Fu, and Z. Li, "Securityconstrained unit commitment with natural gas transmission constraints," IEEE Transactions on Power Systems, vol. 24, no. 3, pp. 1523-1536, 2009.

[14] H. G, L. K-A, Q. E, et al, "Optimization models for the natural gas value chain," in Geometric modelling, numerical simulation, and optimization, Springer, 2007, p. 211-264.

[15] C. Draxl, A. Clifton, B. Hodge, and J. Mccaa, "The Wind Integration National Dataset ( WIND ) Toolkit," Applied Energy, vol. 151, pp. 355-366, 2015. 